\title{
On manifolds with non-negative Ricci curvature and Sobolev inequalities
}

\author{
M. LEDOUX
}

Let $M$ be a complete $n$-dimensional Riemanian manifold with nonnegative Ricci curvature in which one of the Sobolev inequalities $\left(\int|f|^{p} d v\right)^{1 / p} \leq C\left(\int|\nabla f|^{q} d v\right)^{1 / q}, f \in C_{0}^{\infty}(M), 1 \leq q<n, 1 / p=$ $1 / q-1 / n$, is satisfied with $C$ the optimal constant of this inequality in $\mathbb{R}^{n}$. Then $M$ is isometric to $\mathbb{R}^{n}$.

Let $M$ be a complete Riemannian manifold of dimension $n \geq 2$. Denote by $d v$ the Riemannian volume element on $M$ and by $\nabla$ the gradient operator.

In this note, we are concerned with manifolds $M$ in which a Sobolev inequality of the type

$$
\left(\int|f|^{p} d v\right)^{1 / p} \leq C\left(\int|\nabla f|^{q} d v\right)^{1 / q}
$$

$1 \leq q<n, 1 / p=1 / q-1 / n$, holds for some constant $C$ and all $C^{\infty}$ compactly supported functions $f$ on $M$. The best constants $C=K(n, q)$ for which (1) holds in $\mathbb{R}^{n}$ are known and were described by Th. Aubin $[\mathrm{Au}]$ and G. Talenti [Ta]. Namely, $K(n, 1)=n^{-1} \omega_{n}^{-1 / n}$ where $\omega_{n}$ is the volume of the Euclidean unit ball in $\mathbb{R}^{n}$, while

$$
K(n, q)=\frac{1}{n}\left(\frac{n(q-1)}{n-q}\right)^{(q-1) / q}\left(\frac{\Gamma(n+1)}{n \omega_{n} \Gamma(n / q) \Gamma(n+1-n / q)}\right)^{1 / n}
$$

if $q>1$. Moreover, for $q>1$, the equality in (1) is attained by the functions $\left(\lambda+|x|^{q /(q-1)}\right)^{1-(n / q)}, \lambda>0$, where $|x|$ is the Euclidean length of the vector $x$ in $\mathbb{R}^{n}$. We are actually interested here in the geometry of those manifolds $M$ for which one of the Sobolev inequalities (1) is satisfied with the best constant $C=K(n, q)$ of $\mathbb{R}^{n}$. The result of this note is the following theorem.

Theorem. Let $M$ be a complete $n$-dimensional Riemannian manifold with non-negative Ricci curvature. If one of the Sobolev inequalities (1) is satisfied with $C=K(n, q)$, then $M$ is isometric to $\mathbb{R}^{n}$. 
The particular case $q=1(p=n /(n-1))$ is of course well-known. In this case indeed, the Sobolev inequality is equivalent to the isoperimetric inequality

$$
\left(\operatorname{vol}_{n}(\Omega)\right)^{(n-1) / n} \leq K(n, 1) \operatorname{vol}_{n-1}(\partial \Omega)
$$

where $\partial \Omega$ is the boundary of a smooth bounded open set $\Omega$ in $M$. If we let $V\left(x_{0}, s\right)=V(s)$ be the volume of the geodesic ball $B\left(x_{0}, s\right)=B(s)$ with center $x_{0}$ and radius $s$ in $M$, we have

$$
\frac{d}{d s} \operatorname{vol}_{n}(B(s))=\operatorname{vol}_{n-1}(\partial B(s)) .
$$

Hence, setting $\Omega=B(s)$ in the isoperimetric inequality, we get

$$
V(s)^{(n-1) / n} \leq K(n, 1) V^{\prime}(s)
$$

for all $s$. Integrating yields $V(s) \geq(n K(n, 1))^{-n} s^{n}$, and since $K(n, 1)=$ $n^{-1} \omega_{n}^{-1 / n}$, for every $s$,

$$
V(s) \geq V_{0}(s)
$$

where $V_{0}(s)=\omega_{n} s^{n}$ is the volume of the Euclidean ball of radius $s$ in $\mathbb{R}^{n}$. If $M$ has non-negative Ricci curvature, by Bishop's comparison theorem (cf. e.g. [Ch]) $V(s) \leq V_{0}(s)$ for every $s$, and by (2) and the case of equality, $M$ is isometric to $\mathbb{R}^{n}$. The main interest of the Theorem therefore lies in the case $q>1$. As usual, the classical value $q=2$ (and $p=2 n /(n-2)$ ) is of particular interest (see below). It should be noticed that known results already imply that the scalar curvature of $M$ is zero in this case (cf. [He], Prop. 4.10).

Proof of the Theorem. It is inspired by the technique developed in the recent work [B-L] where a sharp bound on the diameter of a compact Riemannian manifold satisfying a Sobolev inequality is obtained, extending the classical Myers theorem.

We thus assume that the Sobolev inequality (1) is satisfied with $C=$ $K(n, q)$ for some $q>1$. Recall first that the extremal functions of this inequality in $\mathbb{R}^{n}$ are the functions $\left(\lambda+|x|^{q^{\prime}}\right)^{1-(n / q)}, \lambda>0$, where $q^{\prime}=$ $q /(q-1)$. Let now $x_{0}$ be a fixed point in $M$ and let $\theta>1$. Set $f=\theta^{-1} d\left(\cdot, x_{0}\right)$ where $d$ is the distance function on $M$. The idea is then to apply the Sobolev inequality (1), with $C=K(n, q)$, to $\left(\lambda+f^{q^{\prime}}\right)^{1-(n / q)}$, for every $\lambda>0$ to deduce a differential inequality whose solutions may be compared to the extremal Euclidean case. Set, for every $\lambda>0$,

$$
F(\lambda)=\frac{1}{n-1} \int \frac{1}{\left(\lambda+f^{q^{\prime}}\right)^{n-1}} d v
$$


Note first that $F$ is well defined and continuously differentiable in $\lambda$. Indeed, by Fubini's theorem, for every $\lambda>0$,

$$
F(\lambda)=q^{\prime} \int_{0}^{\infty} V(\theta s) \frac{s^{q^{\prime}-1}}{\left(\lambda+s^{q^{\prime}}\right)^{n}} d s
$$

(where we recall that $V(s)=V\left(x_{0}, s\right)$ is the volume of the ball with center $x_{0}$ and radius $s$ ). By Bishop's comparison theorem, $V(s) \leq V_{0}(s)$ for every $s$. It follows that $0 \leq F(\lambda)<\infty$ and that $F$ is differentiable.

Together with a simple approximation procedure, apply now the Sobolev inequality (1) with $C=K(n, q)$ to $\left(\lambda+f^{q^{\prime}}\right)^{1-(n / q)}$ for every $\lambda>0$. Since $|\nabla f| \leq 1$ and $1 / p=1 / q-1 / n$, we get

$$
\left(\int \frac{1}{\left(\lambda+f^{q^{\prime}}\right)^{n}} d v\right)^{1 / p} \leq K(n, q)\left(\frac{n-q}{q-1}\right)\left(\int \frac{f^{q^{\prime}}}{\left(\lambda+f^{q^{\prime}}\right)^{n}} d v\right)^{1 / q} .
$$

In other words, setting

$$
\alpha=\left(K(n, q)\left(\frac{n-q}{q-1}\right)\right)^{-q}
$$

for every $\lambda>0$,

$$
\alpha\left(-F^{\prime}(\lambda)\right)^{q / p}-\lambda F^{\prime}(\lambda) \leq(n-1) F(\lambda) .
$$

We now compare the solutions of the differential inequality (3) to the solutions $H$ of the differential equality

$$
\alpha\left(-H^{\prime}(\lambda)\right)^{q / p}-\lambda H^{\prime}(\lambda)=(n-1) H(\lambda), \quad \lambda>0 .
$$

It is plain that a particular solution $H_{0}$ of (4) is given by the extremal functions of the Sobolev inequality in $\mathbb{R}^{n}$, namely

$$
H_{0}(\lambda)=\frac{1}{n-1} \int_{\mathbb{R}^{n}} \frac{1}{\left(\lambda+|x|^{q^{\prime}}\right)^{n-1}} d x=\frac{A}{\lambda^{(n / q)-1}}
$$

where

$$
A=H_{0}(1)=\frac{1}{n-1} \int_{\mathbb{R}^{n}} \frac{1}{\left.\left(1+|x| q^{\prime}\right)\right)^{n-1}} d x=\frac{q}{n-q}\left(\frac{\alpha(n-q)}{n(q-1)}\right)^{p /(p-q)}
$$

(as a solution of (4)). 
We claim that if $F\left(\lambda_{0}\right)<H_{0}\left(\lambda_{0}\right)$ for some $\lambda_{0}>0$, then $F(\lambda)<H_{0}(\lambda)$ for every $\lambda \leq \lambda_{0}$. Indeed, if this is not the case, let $\lambda_{1}$ be defined by

$$
\lambda_{1}=\sup \left\{\lambda<\lambda_{0} ; F(\lambda)=H_{0}(\lambda)\right\} .
$$

Now, for every $\lambda>0, \varphi_{\lambda}(X)=\alpha X^{q / p}+\lambda X$ is strictly increasing in $X \geq 0$ so that (3) reads as

$$
-F^{\prime}(\lambda) \leq \varphi_{\lambda}^{-1}((n-1) F(\lambda))
$$

while, by (4),

$$
-H_{0}^{\prime}(\lambda)=\varphi_{\lambda}^{-1}\left((n-1) H_{0}(\lambda)\right)
$$

Therefore

$$
\left(F-H_{0}\right)^{\prime}(\lambda) \geq \varphi_{\lambda}^{-1}\left((n-1) H_{0}(\lambda)\right)-\varphi_{\lambda}^{-1}((n-1) F(\lambda)) \geq 0
$$

on the set $\left\{F \leq H_{0}\right\}$. Hence $\left(F-H_{0}\right)^{\prime} \geq 0$ on the interval $\left[\lambda_{1}, \lambda_{0}\right]$ so that $F-H_{0}$ is non-decreasing on this interval. But then, in particular,

$$
0=\left(F-H_{0}\right)\left(\lambda_{1}\right) \leq\left(F-H_{0}\right)\left(\lambda_{0}\right)<0
$$

which is a contradiction.

Recall now, $\lambda>0$,

$$
F(\lambda)=\frac{1}{n-1} \int \frac{1}{\left(\lambda+f^{q^{\prime}}\right)^{n-1}} d v=q^{\prime} \int_{0}^{\infty} V(\theta s) \frac{s^{q^{\prime}-1}}{\left(\lambda+s^{q^{\prime}}\right)^{n}} d s
$$

while

$$
\begin{aligned}
H_{0}(\lambda) & =\frac{1}{n-1} \int_{\mathbb{R}^{n}} \frac{1}{\left(\lambda+|x|^{q^{\prime}}\right)^{n-1}} d x=q^{\prime} \int_{0}^{\infty} V_{0}(s) \frac{s^{q^{\prime}-1}}{\left(\lambda+s^{q^{\prime}}\right)^{n}} d s \\
& =\frac{A}{\lambda^{(n / q)-1}} .
\end{aligned}
$$

The local geometry indicates that

$$
\liminf _{\lambda \rightarrow 0} \frac{F(\lambda)}{H_{0}(\lambda)} \geq \theta^{n}>1
$$

Indeed, write

$$
F(\lambda)=q^{\prime} \theta^{(n-1) q^{\prime}} \int_{0}^{\infty} V(s) \frac{s^{q^{\prime}-1}}{\left(\theta^{q^{\prime}} \lambda+s^{q^{\prime}}\right)^{n}} d s
$$


As $V(s) \sim V_{0}(s)$ when $s \rightarrow 0$, for every $\varepsilon>0$, there is $\delta>0$ such that, for every $\lambda>0$,

$$
\begin{aligned}
\int_{0}^{\infty} V(s) \frac{s^{q^{\prime}-1}}{\left(\theta^{q^{\prime}} \lambda+s^{q^{\prime}}\right)^{n}} d s & \geq(1-\varepsilon) \int_{0}^{\delta} V_{0}(s) \frac{s^{q^{\prime}-1}}{\left(\theta^{q^{\prime}} \lambda+s^{q^{\prime}}\right)^{n}} d s \\
& \geq \frac{(1-\varepsilon)}{\theta^{q^{\prime}((n / q)-1)} \lambda^{(n / q)-1}} \int_{0}^{\delta / \theta \lambda^{1 / q^{\prime}}} V_{0}(s) \frac{s^{q^{\prime}-1}}{\left(1+s^{q^{\prime}}\right)^{n}} d s .
\end{aligned}
$$

Hence, for every $\lambda>0$,

$$
\frac{F(\lambda)}{H_{0}(\lambda)} \geq \theta^{n} \frac{(1-\varepsilon) \int_{0}^{\delta / \theta \lambda^{1 / q^{\prime}}} V_{0}(s) \frac{s^{q^{\prime}-1}}{\left(1+s^{q^{\prime}}\right)^{n}} d s}{\int_{0}^{\infty} V_{0}(s) \frac{s^{q^{\prime}-1}}{\left(1+s^{q^{\prime}}\right)^{n}} d s}
$$

from which (5) follows as $\lambda \rightarrow 0$.

We can now conclude the proof of the Theorem. By the claim and (5), we have that $F(\lambda) \geq H_{0}(\lambda)$ for every $\lambda>0$, that is

$$
\int_{0}^{\infty}\left[V(\theta s)-V_{0}(s)\right] \frac{s^{q^{\prime}-1}}{\left(\lambda+s^{q^{\prime}}\right)^{n}} d s \geq 0 .
$$

Letting $\theta \rightarrow 1$,

$$
\int_{0}^{\infty}\left[V(s)-V_{0}(s)\right] \frac{s^{q^{\prime}-1}}{\left(\lambda+s^{q^{\prime}}\right)^{n}} d s \geq 0
$$

for every $\lambda>0$. Since by Bishop's theorem $V(s) \leq V_{0}(s)$ for every $s$ when $M$ has non-negative curvature, it must be that $V(s)=V_{0}(s)$ for almost every $s$, and thus every $s$ by continuity. By the case of equality in Bishop's theorem, $M$ is isometric to $\mathbb{R}^{n}$. The proof of the Theorem is complete.

It is natural to conjecture that the Theorem may actually be turned into a volume comparison statement as it is the case for $q=1$. That is, in a manifold $M$ satisfying the Sobolev inequality (1) with the constant $K(n, q)$ for some $q>1$, and without any curvature assumption, for every $x_{0}$ and every $s$,

$$
V\left(x_{0}, s\right) \geq V_{0}(s)
$$

This is well-known up to a constant (depending only on $n$ and $q$ ) but the preceding proof does not seem to be able to yield such a conclusion. 
To conclude this note, we comment some related comparison theorem. The Sobolev inequality (1) belongs to a general family of inequalities of the type

$$
\left(\int|f|^{r} d v\right)^{1 / r} \leq C\left(\int|f|^{s} d v\right)^{\theta / s}\left(\int|\nabla f|^{q} d v\right)^{(1-\theta) / q}, \quad f \in C_{0}^{\infty}(M),
$$

with

$$
\frac{1}{r}=\frac{\theta}{s}+\frac{1-\theta}{p}
$$

(cf. [B-C-L-SC]). Inequality (1) corresponds to $\theta=0$. When $q=2$, the classical value, and $r=2$, other choices of interest are $\theta=2 /(n+2)$ which corresponds to the Nash inequality

$$
\left(\int|f|^{2} d v\right)^{1+(2 / n)} \leq C\left(\int|f| d v\right)^{4 / n} \int|\nabla f|^{2} d v, \quad f \in C_{0}^{\infty}(M),
$$

and the limiting value $\theta=1$ which corresponds to the logarithmic Sobolev or entropy-energy inequality

$$
\int f^{2} \log f^{2} d v \leq \frac{n}{2} \log \left(C \int|\nabla f|^{2} d v\right), \quad f \in C_{0}^{\infty}(M), \quad \int f^{2} d v=1
$$

(cf. [Da]). As for the Sobolev inequality (1), the optimal constants for these two inequalities (6) and (7) in $\mathbb{R}^{n}$ are known ([C-L] and [Ca] respectively), so that one may ask for a statement analogous to the Theorem in case of these inequalities. As a result, it was shown in [B-C-L] that this is indeed the case for the logarithmic Sobolev inequality (7), that is, a $n$-dimensional Riemannian manifold with non-negative Ricci curvature satisfying (7) with the best constant of $\mathbb{R}^{n}$ is isometric to $\mathbb{R}^{n}$. The proof there relies on optimal heat kernel bounds in manifolds satisfying the logarithmic Sobolev inequality (7) with the best constant of $\mathbb{R}^{n}$. Namely, if $p_{t}(x, y)$ denotes the heat kernel on $M$, then, for every $t>0$,

$$
\sup _{x, y \in M} p_{t}(x, y) \leq \frac{1}{(4 \pi t)^{n / 2}}=\sup _{x, y \in \mathbb{R}^{n}} p_{t}^{0}(x, y)
$$

where $p_{t}^{0}(x, y)$ is the heat kernel on $\mathbb{R}^{n}$. One then concludes with the results of $\mathrm{P} . \mathrm{Li}[\mathrm{Li}]$ relating an optimal large time heat kernel decay to the maximal volume growth of balls in manifolds with non-negative Ricci curvature. The analogous results for the Nash inequality (6) are so far open. 


\section{References.}

[Au] Th. Aubin, Nonlinear analysis on manifolds, Monge-Ampère equations, Springer, 1982.

[B-C-L] D. Bakry, D. Concordet and M. Ledoux, Optimal heat kernel bounds under logarithmic Sobolev inequalities. ESAIM: Probability and Statistics 1 (1997), 391-407.

[B-C-L-SC] D. Bakry, T. Coulhon, M. Ledoux and L. Saloff-Coste, Sobolev inequalities in disguise, Indiana J. Math. 44 (1995), 1033-1074.

[B-L] D. Bakry and M. Ledoux, Sobolev inequalities and Myers's diameter theorem for an abstract Markov generator, Duke Math. J. 85 (1996), 253-270.

[Ca] E. Carlen, Superadditivity of Fisher's information and logarithmic Sobolev inequalities, J. Funct. Anal. 101 (1991), 194-211.

[C-L] E. Carlen and M. Loss, Sharp constant in Nash's inequality, Duke Math. J., International Math. Research Notices 7 (1993), 213-215.

[Ch] I. Chavel, Riemannian geometry: a modern introduction, Cambridge Univ. Press, 1993.

[Da] E.B. Davies, Heat kernels and spectral theory, Cambridge Univ. Press, 1989.

[He] E. Hebey, Sobolev spaces on Riemannian manifolds, Lecture Notes in Math. 1635, Springer, 1996.

[Li] P. Li, Large time behavior of the heat equation on complete manifolds with non-negative Ricci curvature, Ann. Math. 124 (1986), 1-21.

[Ta] G. Talenti, Best constants in Sobolev inequality, Ann. di Matem. Pura ed Appl. 110 (1976), 353-372.

Received March 6, 1997.

Laboratoire de Statistique et Probabilités associé au C.N.R.S., UNIVERSité PAUL-SABATIER, 31062, TOULOUSE (FRANCE)

E-mail address: ledoux@cict.fr 